\title{
Observation of Coupling between Vibrational and Configurational Entropy in a Supercooled Molecular Chalcogenide Glass-Forming Liquid
}

Sabyasachi Sen ${ }^{1}$ and Bruce G. Aitken ${ }^{2}$, 


\begin{abstract}
The temperature dependence of the line widths and frequencies of the vibrational modes of $\mathrm{As}_{4} \mathrm{~S}_{3}$ molecules is studied in the $\mathrm{Ge}_{3} \mathrm{As}_{52} \mathrm{~S}_{45}$ molecular glass and supercooled liquid at temperatures ranging from $\sim T_{g}-189 \mathrm{~K}$ to up to $\sim T_{g}+111 \mathrm{~K}$, using Raman spectroscopy. The results reveal the presence of strong dynamical coupling between vibrational and configurational degrees of freedom of the molecules such that both the phonon lifetime and the density of states are affected by the rotational (and possibly translational) molecular dynamics in the supercooled liquid. The physical origin of such coupling is discussed within the framework of the potential energy landscape model of the glass transition.
\end{abstract}

Keywords: vibrational entropy; configurational entropy; vibration-rotation coupling; potential energy landscape; molecular chalcogenide; Raman spectroscopy 


\section{Introduction}

It is widely accepted that the heat capacity jump $\Delta \mathrm{C}_{\mathrm{p}}$ across the glass transition is associated with excess entropy $S_{\text {exc }}$ in the supercooled liquid that is primarily configurational in nature, i.e. $S_{\text {conf }} \approx S_{\text {exc }}$, as the vibrational entropy $S_{\text {vib }}$ reaches the Dulong-Petit limit of $3 R / g$ atom in the glassy state below the glass transition temperature $T_{g}[1-4]$. In this scenario, $\mathrm{S}_{\mathrm{vib}}$ and $\mathrm{S}_{\text {conf }}$ can be treated essentially as decoupled quantities. Experimental estimates of the relative contributions of $S_{\text {vib }}$ and $S_{\text {conf }}$ to $S_{\text {exc }}$ associated with the glass transition are few and far between. These estimates made in previous studies on amorphous chalcogenides such as $\mathrm{Se}$ and $\mathrm{GeSe}_{4}$ indicated that the approximation $\mathrm{S}_{\mathrm{conf}} \approx \mathrm{S}_{\mathrm{exc}}$ may not be universally valid and $\mathrm{S}_{\mathrm{vib}}$ may contribute significantly to $\mathrm{S}_{\mathrm{exc}}$ near and above $T_{g}[5,6]$. It may be noted that knowledge of the relative magnitudes of $S_{\text {vib }}$ and $S_{\text {conf }}$ and their degree of coupling is important in understanding the nature of the potential energy landscape (PEL) in a supercooled liquid [7-10]. This situation is particularly relevant for molecular liquids where individual molecules have vibrational and configurational (rotational and translational) degrees of freedom. Here we report the results of a variable-temperature Raman spectroscopic study of a molecular chalcogenide in glassy and supercooled liquid states to demonstrate significant coupling between the vibrational and configurational dynamics of the constituent molecules at $\mathrm{T}>T_{g}$. The implications of these results on our current understanding of the glass transition within the PEL framework are discussed. 


\section{Experimental}

\subsection{Sample Preparation}

The molecular chalcogenide glass of composition $\mathrm{Ge}_{3} \mathrm{As}_{52} \mathrm{~S}_{45}$ is chosen for this study. This glass was shown in previous studies to consist of globular $\mathrm{As}_{4} \mathrm{~S}_{3}$ cage molecules with strong intramolecular covalent bonding that are held together by weak van der Waals forces [11-14]. The glass was synthesized by melting a mixture of the constituent elements $(\geq 99.9995 \%$ purity, metals basis) in an evacuated (10 $10^{-6}$ Torr) fused silica ampoule and subsequently quenching the ampoule in water. The small amount of Ge doping is necessary in stabilizing the glass against crystallization. The $T_{g}$ of this glass was reported to be $312 \mathrm{~K}$ [11].

\subsection{Raman Spectroscopy}

Raman spectra were collected over a temperature range of $123 \mathrm{~K}$ to $423 \mathrm{~K}$ using a Bruker RFS 100/S Fourier-transform (FT) Raman spectrometer equipped with a confocal microscope (Olympus BX51M) and a frequency-doubled Nd:YAG laser operating at a wavelength of 1064 nm. Glass samples of dimensions $\sim 1 \mathrm{x} 1 \mathrm{~mm}$ and thicknesses of $\sim 100 \mu \mathrm{m}$ were heated on a commercial cryostat / hot stage (THMS600, Linkam Scientific Instruments Ltd.). Temperature was controlled using a temperature programmer and power supply (TMS94) connected to the stage. Cooling below ambient temperature was achieved by flowing liquid nitrogen through the cell. The upper limit of the temperature range for these experiments was set at $423 \mathrm{~K}$ as the supercooled liquid undergoes rapid crystallization above this temperature while the lower limit was set by the instrumental limit of the sample stage. Temperature calibration of the system was performed in separate experiments using known phase transitions in crystalline standards. A 
long working distance objective (Olympus LMPlanFL, magnification 20x, numerical aperture 0.4 , working distance $12 \mathrm{~mm}$ ) was used to focus the laser light on the sample, at a power level of $30 \mathrm{~mW}$. The sample was equilibrated at each temperature for several minutes before data collection. Raman spectra were obtained at each temperature with a resolution of $1 \mathrm{~cm}^{-1}$ and each spectrum represents an average of about 100 scans $(\sim 5 \mathrm{~min})$. Data were collected during both heating and cooling to ensure complete reversibility.

\section{Results}

The temperature dependence of the unpolarized Raman spectra of the $\mathrm{Ge}_{3} \mathrm{As}_{52} \mathrm{~S}_{45}$ glass and supercooled liquid is shown in Fig. 1. Five main vibrational bands in these spectra are distinguishable: a group of one broad and two narrow bands of relatively low intensity centered at 178,202 and $218 \mathrm{~cm}^{-1}$, respectively; a relatively narrow and intense band at $275 \mathrm{~cm}^{-1}$ and a relatively broad and moderately intense band centered at $355 \mathrm{~cm}^{-1}$ (Fig. 1). In addition, the band at $178 \mathrm{~cm}^{-1}$ has a weak shoulder near $172 \mathrm{~cm}^{-1}$. These vibrational bands can all be assigned to various intramolecular vibrational modes of the $\mathrm{As}_{4} \mathrm{~S}_{3}$ molecule [11,15-18]. In previous studies of crystalline $\mathrm{As}_{4} \mathrm{~S}_{3}[17,18]$ the band at $178 \mathrm{~cm}^{-1}$ was assigned to the $\mathrm{S}-\mathrm{As}-\mathrm{S}$ bending mode of the apical $\mathrm{AsS}_{3}$ pyramid in the molecule, while that at $218 \mathrm{~cm}^{-1}$ was linked to the breathing mode of this pyramid which involves opposing motion of the $3 \mathrm{~S}$ atoms with respect to the top As atom. On the other hand the bands at 202 and $275 \mathrm{~cm}^{-1}$ correspond to antisymmetric and symmetric breathing of the $\mathrm{As}_{3}$ triangle at the base of the molecule. Finally, the band near 355 $\mathrm{cm}^{-1}$ arises from symmetric stretching of the As-S bonds involving the basal As atoms and may also contain contribution from the stretching of As-S bonds involving the apical As atom. In crystalline $\mathrm{As}_{4} \mathrm{~S}_{3}$ these two stretching modes appear as separate bands near 370 and $340 \mathrm{~cm}^{-1}$, 
respectively. Finally, this band is also likely to have a non-negligible contribution from the symmetric stretching of $\mathrm{GeS}_{4}$ tetrahedra (characteristic frequency $\sim 345 \mathrm{~cm}^{-1}$ in Ge-As-S glasses), that are known to be present in this glass, albeit in small concentration [19,20].

The position and width of the band at $218 \mathrm{~cm}^{-1}$, as well as the position of the composite band at $178 \mathrm{~cm}^{-1}$, vary insignificantly with temperature, within the instrumental resolution. Therefore, in this study we focus on the temperature dependent behavior of the bands at 202, 275 and $355 \mathrm{~cm}^{-1}$. The temperature dependence of the line widths of these modes is shown in Fig. 2 and that of the frequencies is shown in Fig. 3. While the width of the band at $202 \mathrm{~cm}^{-1}$ does not change significantly with temperature, that of the $275 \mathrm{~cm}^{-1}$ band shows a linear increase with temperature at a rate of $\sim 0.0029 \mathrm{~cm}^{-1} / \mathrm{K}$ (Fig. 2). However, the most dramatic temperature dependence is observed for the $355 \mathrm{~cm}^{-1}$ band which increases in width at a rate of $\sim 0.0087$ $\mathrm{cm}^{-1} / \mathrm{K}$ below $T_{g}$ and at a rate of $\sim 0.0275 \mathrm{~cm}^{-1} / \mathrm{K}$ above $T_{g}$ (Fig. 2).

The positions of the 202 and $275 \mathrm{~cm}^{-1}$ bands shift linearly to lower frequency at higher temperatures at a rate of $-0.012 \mathrm{~cm}^{-1} / \mathrm{K}$ over the entire temperature range, indicating thermal mode softening (Fig. 3). It is interesting to note that the softening rate for these intramolecular vibrational modes is comparable to previously reported thermal softening rates of Ge-Se and SeSe stretching modes in binary Ge-selenide liquids immediately above $T_{g}[6]$. On the other hand, the temperature dependence of the position of the $355 \mathrm{~cm}^{-1}$ band displays an unusual behaviorthe usual mode softening is observed as the temperature is increased up to $T_{g}$, however, in the supercooled liquid state at $\mathrm{T}>T_{g}$ a frequency hardening of this mode is observed as the frequency increases with increasing temperature (Fig. 3). The rates of frequency softening and hardening are nearly equal within error, with magnitude ranging between 0.008 and 0.010 $\mathrm{cm}^{-1} / \mathrm{K}$. It may be noted here that breaks across glass transition in the slopes of frequency, 
linewidth and relative intensity of Raman bands with temperature have been reported in previous studies for amorphous silicate networks, where such changes were related to the anharmonic vibrational effects and to the onset of structural and configurational changes in the supercooled liquid [21,22].

\section{Discussion}

Line broadening and thermal mode softening of vibrational modes typically result from anharmonic effects that are also responsible for thermal expansion [23]. However, the temperature dependent behavior of the intramolecular modes of the $\mathrm{As}_{4} \mathrm{~S}_{3}$ molecules in the $\mathrm{Ge}_{3} \mathrm{As}_{52} \mathrm{~S}_{45}$ glass and supercooled liquid is not entirely consistent with anharmonic effects. For example, such effects are expected to be stronger for the lower-frequency bending modes than for higher-frequency stretching modes, yet the frequency of the S-As-S bending mode at 178 $\mathrm{cm}^{-1}$ does not display any significant change with temperature (Fig. 1). Moreover, although the bands at 202 and $275 \mathrm{~cm}^{-1}$, corresponding to the antisymmetric and symmetric breathing of the basal $\mathrm{As}_{3}$ triangle, involve the same atoms and show the same rate of thermal softening, the line width of the former band is practically independent of temperature while that of the latter band increases linearly (Figs. 2, 3). Finally, the transition from the glass to supercooled liquid state is expected to be accompanied by an increase in the thermal expansion coefficient which would be expected to give rise to an increased rate of mode softening in the supercooled liquid state. However, the mode softening rate for the 202 and $275 \mathrm{~cm}^{-1}$ bands does not display any discernible change across $T_{g}$ (Fig. 3). Additionally, the $355 \mathrm{~cm}^{-1}$ band displays a frequency hardening behavior with temperature above the glass transition which clearly cannot be explained by anharmonicity effects. 
It was demonstrated in a previous study based on nuclear magnetic resonance spectroscopy [14] that the constituent $\mathrm{As}_{4} \mathrm{~S}_{3}$ cage molecules in the supercooled $\mathrm{Ge}_{3} \mathrm{As}_{52} \mathrm{~S}_{45}$ liquid undergo rapid isotropic rotational reorientation above $T_{g}$ that is temporally decoupled from the timescale of structural relaxation by several orders of magnitude. Furthermore, such motion continues well into the glassy state. The quasi-continuum of these rotational excitations can couple to the vibrational modes and result into a lowering of the lifetime of the latter and equivalently a line broadening of the bands in the Raman spectra. Therefore, it is likely that the temperature induced line broadening of the intramolecular vibrational modes observed in this study is primarily a manifestation of the decreasing lifetime of the phonons resulting from coupling of these phonons with the molecular rotational motion. In fact, such spectral line broadening mechanisms were also proposed in the literature for the molecular crystal $\mathrm{C}_{60}$ where the spherical $\mathrm{C}_{60}$ molecules perform rotational motion at their respective lattice sites [24].

In this scenario, the line widths of the Raman bands are a function of both the available rotational and vibrational density of states at any temperature and the degree of vibration-rotation coupling. The latter depends on the magnitude of the matrix elements representing the vibrationrotation coupling and consequently on the symmetry of the vibrational mode. Therefore, the increasing line width with temperature for the 355 and $275 \mathrm{~cm}^{-1}$ bands (Fig. 2) is consistent with the increasing density of the available rotational states for the $\mathrm{As}_{4} \mathrm{~S}_{3}$ molecules. On the other hand, the stronger temperature dependence of the line width of the $355 \mathrm{~cm}^{-1}$ band compared to that for the $275 \mathrm{~cm}^{-1}$ band suggests a stronger vibration-rotation coupling for the former vibrational mode. Hence, the line broadening rate of the $355 \mathrm{~cm}^{-1}$ band serves as a sensitive measure of the temperature dependence of the molecular rotational density of states in this glass/supercooled liquid. The abrupt increase in the line broadening rate for this band across $T_{g}$ 
then implies sudden availability of an excess of rotational states as the glass transitions into the supercooled liquid state. This hypothesis is consistent with the PEL model of glass transition in liquids where cooling results in an abrupt decrease in the number of metabasins or inherent structural configurations accessible to the supercooled liquid in the energy landscape [9,25]. Consequently, the rotational states within the metabasins that contribute to $\mathrm{S}_{\text {conf }}$ at $\mathrm{T}>T_{g}$ become unavailable to the molecules as those metabasins become inaccessible below the glass transition $[10]$.

As discussed in detail by Zallen and Slade [23], the temperature dependence of the phonon frequencies in vibrational spectra at constant pressure $(\mathrm{d} v / \mathrm{dT})_{\mathrm{P}}$ has two contributions that originate from the anharmonicity of potential wells. These are: an "implicit" contribution $(\mathrm{d} v / \mathrm{dP})_{\mathrm{T}}$ from volume change due to thermal expansion and an "explicit" dynamic contribution $(\mathrm{d} v / \mathrm{dT})_{\mathrm{V}}$ from change in vibrational amplitude of atoms at constant volume i.e. at fixed equilibrium positions. The implicit contribution arises from a quasi-harmonic approach used to analyze thermal expansion effects that does not consider the quantized nature of the phonon excitations. In contrast, the explicit term represents "intrinsic" mode anharmonicity that is a result of phonon-phonon scattering interactions [26]. The relationship between these quantities can be expressed as [23]:

$$
(\mathrm{d} v / \mathrm{dT})_{\mathrm{P}}=(\mathrm{d} v / \mathrm{dT})_{\mathrm{V}}-(\alpha / \beta)(\mathrm{d} v / \mathrm{dP})_{\mathrm{T}}
$$

where, $\alpha$ and $\beta$ are volume thermal expansion coefficient and compressibility, respectively. Raman spectroscopic measurements at variable pressure and temperature show that $(\mathrm{d} v / \mathrm{dT})_{\mathrm{P}}$ for internal or intramolecular vibrational modes in molecular chalcogenides such as $\mathrm{As}_{4} \mathrm{~S}_{4}$ is 
dominated by the explicit effect. The values of $\alpha, \beta$ and $(\mathrm{dv} / \mathrm{dP})_{\mathrm{T}}$ for the $275 \mathrm{~cm}^{-1}$ band are reported in previous studies for the $\mathrm{Ge}_{3} \mathrm{As}_{52} \mathrm{~S}_{45}$ glass $[11,27]$ to be $2.87 \mathrm{e}-4 \mathrm{~K}^{-1}, 19.4 \mathrm{GPa}^{-1}$ and $0.93 \mathrm{~cm}^{-1} \mathrm{GPa}^{-1}$, respectively, which yield the value of $-0.005 \mathrm{~cm}^{-1} \mathrm{~K}^{-1}$ for the implicit contribution. When compared with the $(\mathrm{d} v / \mathrm{dT})_{\mathrm{P}}$ for this mode observed in this study $(-0.012$ $\mathrm{cm}^{-1} \mathrm{~K}^{-1}$ ), it is clear that both the implicit and the explicit terms contribute nearly equally towards the frequency softening in the glassy state. The absence of any effect of the glass transition on the $(\mathrm{dv} / \mathrm{dT})_{\mathrm{P}}$ for the 202 and $275 \mathrm{~cm}^{-1}$ bands indicate that the relative contributions of the implicit and explicit terms in Eqn. 1 do not change significantly with temperature.

The frequency softening of the $355 \mathrm{~cm}^{-1}$ band with temperature in the glassy state can be explained in a similar manner, although it should be noted that $(\mathrm{d} v / \mathrm{dP})_{\mathrm{T}}$ for this band was found in a previous study [27] to be negative. This apparently anomalous result was ascribed to the strong sensitivity of this vibrational mode to intermolecular coupling that increases with pressure. Consequently, Eqn. 1 implies that the observed softening behavior in the glassy state is related to the dominant effect of the explicit term $(\mathrm{dv} / \mathrm{dT})_{\mathrm{V}}$. The abrupt switching of the sign of $(\mathrm{d} v / \mathrm{dT})_{\mathrm{P}}$ across the glass transition and frequency hardening in the supercooled liquid state is rather intriguing and suggests a sudden increase in the contribution from the implicit term as the strength of intermolecular coupling changes across glass transition. A similar observation was made in the case of the molecular crystal $\mathrm{C}_{60}$ where increasing intermolecular coupling arising from rapid molecular rotation was suggested to be responsible for temperature induced frequency hardening of some internal modes in the high-temperature fcc phase [24]. It may be noted that, in addition to rapid molecular rotation, the onset of translational diffusion at $\mathrm{T}>T_{g}$ may also be responsible for the increasing rotation-vibration coupling in the supercooled $\mathrm{Ge}_{3} \mathrm{As}_{52} \mathrm{~S}_{45}$ liquid. This hypothesis is consistent with the temperature dependent line broadening behavior of the 355 
$\mathrm{cm}^{-1}$ band as discussed above, which was linked to the availability of an excess of rotational states at $\mathrm{T}>T_{g}$.

\section{Conclusions}

The temperature dependence of the phonon lifetimes and frequencies of the intramolecular vibrational modes indicate significant coupling between the vibrational and configurational degrees of freedom of the constituent $\mathrm{As}_{4} \mathrm{~S}_{3}$ molecules in the $\mathrm{Ge}_{3} \mathrm{As}_{52} \mathrm{~S}_{45}$ glass and supercooled liquid. In particular, an excess of rotational states as well as translational degrees of freedom become available to these molecules at $\mathrm{T}>T_{g}$, which affects the vibrational density of states. Consequently, the vibrational and configurational contributions to the total entropy of a supercooled molecular liquid are temperature dependent and coupled quantities.

\section{Acknowledgements}

This work was supported by a grant from the National Science Foundation (NSF-DMR 1505185) to SS. 


\section{References:}

1. B.O. Mysen, P. Richet, Silicate glasses and melts: properties and structure. Vol. 10. 2005: Elsevier.

2. J.C. Dyre, Rev. Mod. Phys. 78, (2006) 953.

3. G. Adam, J.H. Gibbs, J. Chem. Phys. 43 (1965) 139.

4. C. A. Angell, S. Borick, J. Non-Cryst. Solids 307 (2002) 393.

5. W.A. Phillips, U. Buchenau, N. Nücker, A.J. Dianoux, W. Petry, Phys. Rev. Lett. 63 (1989) 2381.

6. E.L. Gjersing, S. Sen, B.G. Aitken, J. Non-Cryst. Solids 355 (2009) 748.

7. S. Takahara, O. Yamamuro, T. Matsuo, J. Phys. Chem. 99 (1995) 1602.

8. S. Takahara, O. Yamamuro, T. Matsuo, J. Phys. Chem. 99 (1995) 9580.

9. M. Goldstein, J. Chem. Phys. 51 (1969) 3728.

10. G.P. Johari, J. Chem. Phys. 116 (2002) 2043.

11. B.G. Aitken, J. Non-Cryst. Solids 345-346 (2004) 1.

12. S. Sen, S. Gaudio, B.G. Aitken, C.E. Lesher, Phys. Rev. Lett. 97 (2006) 025504.

13. A.C. Wright, B.G. Aitken, G.J. Cuello, J.W. Taylor, J. Non-Cryst. Solids 357 (2011) 2502.

14. E.L. Gjersing, S. Sen, P. Yu, B.G. Aitken, Phys. Rev. B 76 (2007) 214202.

15. B.H. Christian, R.J. Gillespie, J.F. Sawyer, Inorg. Chem. 20 (1981) 3410.

16. T. Chattopadhyay, C. Carlone, A. Jayaraman, H.G.v. Schnering, J. Phys. Chem. Solids 43 (1982) 277.

17. H.J. Whitfield, Aust. J. Chem. 24 (1971) 697.

18. W. Bues, M. Somer, W. Brockner, Z. Naturforsch. 35b (1980) 1063. 
19. B.G. Aitken, C.W. Ponader, J. Non-Cryst. Solids 274 (2000) 124.

20. S. Soyer-Uzun, S. Sen, B.G. Aitken, J. Phys. Chem. C, 113 (2009) 6231.

21. P.F. McMillan, G.H. Wolf, B.T. Poe, Chem. Geol. 96 (1992) 351.

22. P.F. McMillan, B.T. Poe, Ph. Gillet, B. Reynard, Geochim. Cosmochim. Acta, 58 (1994) 3653.

23. R. Zallen, M.L. Slade, Phys. Rev. B18 (1978) 5775.

24. P.H.M. van Loosdrecht, P.J.M. van Bentum, G. Meijer, Phys. Rev. Lett. 68 (1992) 1176.

25. F.H. Stillinger, Energy Landscapes, Inherent Structures, and Condensed-Matter Phenomena, Princeton University Press, Princeton, New Jersey (2015).

26. D.C. Wallace, Thermodynamics of Crystals, Wiley, New York (1972).

27. B. Kalkan, C. Sonneville, C. Martinet, B. Champagnon, B. G. Aitken, S. M. Clark, S. Sen, J. Chem. Phys. 137 (2012) 224503. 


\section{Figure Captions:}

Fig. 1. (a) Unpolarized Raman spectra of $\mathrm{Ge}_{3} \mathrm{As}_{52} \mathrm{~S}_{45}$ glass and supercooled liquid at select temperatures shown alongside each spectrum. Spectra are vertically offset for clarity. All spectra were corrected for the Bose-Einstein population factor and normalized to the intensity of the band at $275 \mathrm{~cm}^{-1}$. (b), (c) and (d) are stack plots of unpolarized Raman spectra with magnified views in different frequency regions displaying temperature dependence of frequency and linewidth. Spectra from bottom to top correspond to temperatures $123 \mathrm{~K}, 173 \mathrm{~K}, 223 \mathrm{~K}$, 293K, 323K, 348K, 373K, 398K and 423K.

Fig. 2. Temperature dependence of the full width at half maximum for (a) $202 \mathrm{~cm}^{-1}$ (filled squares) and $275 \mathrm{~cm}^{-1}$ (open squares) bands and (b) for $355 \mathrm{~cm}^{-1}$ band. The location of the glass transition temperature $T_{g}$ is shown in each plot with an arrow. Straight lines through the data points in (a) are linear least squares fits.

Fig. 3. Temperature dependence of the peak positions for (a) $202 \mathrm{~cm}^{-1}$, (b) $275 \mathrm{~cm}^{-1}$, and (c) $355 \mathrm{~cm}^{-1}$ bands. The location of the glass transition temperature $T_{g}$ is shown in each plot with an arrow. Straight lines through the data points in (a) and (b) are linear least squares fits. 

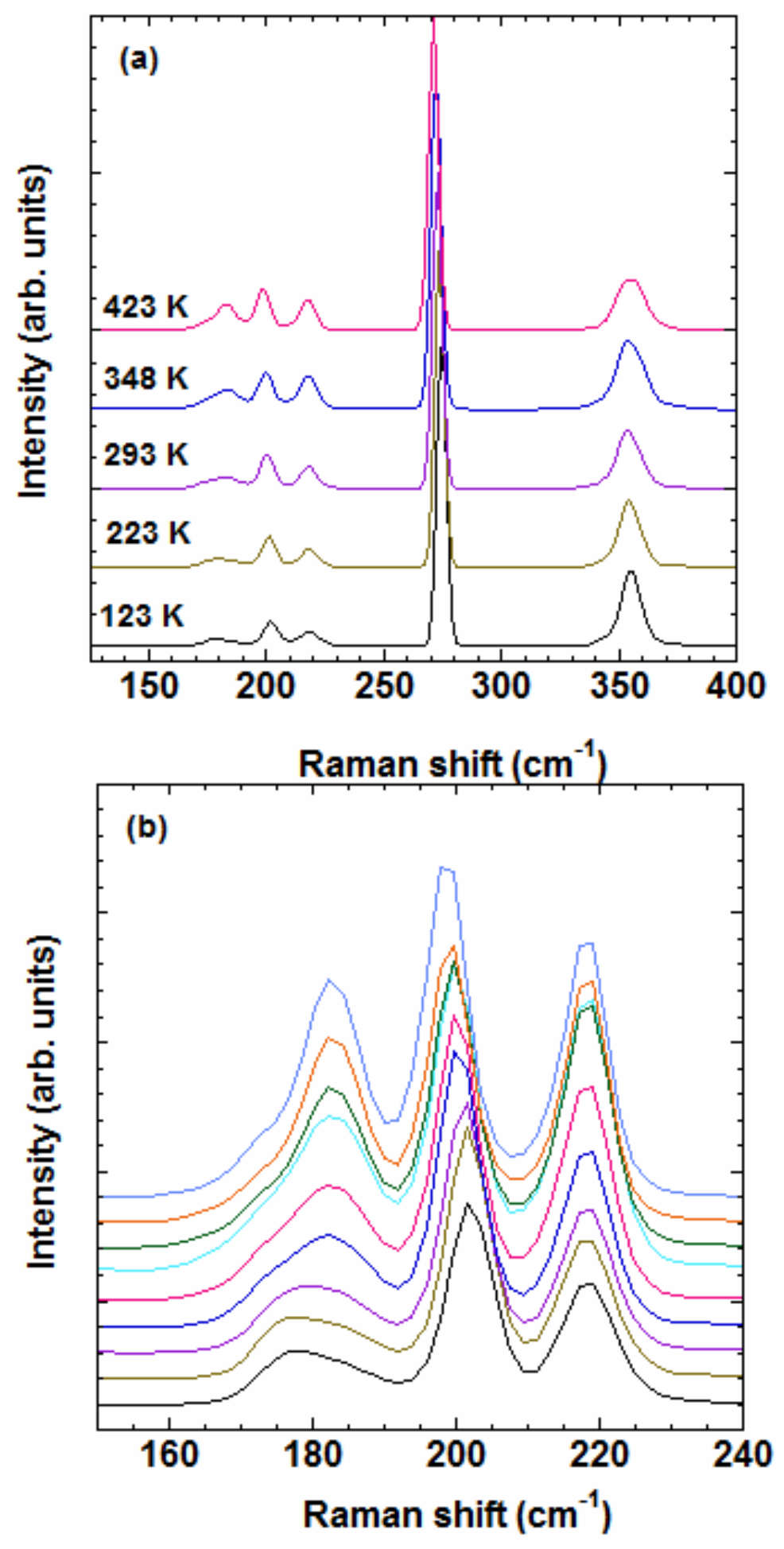

Figure 1 

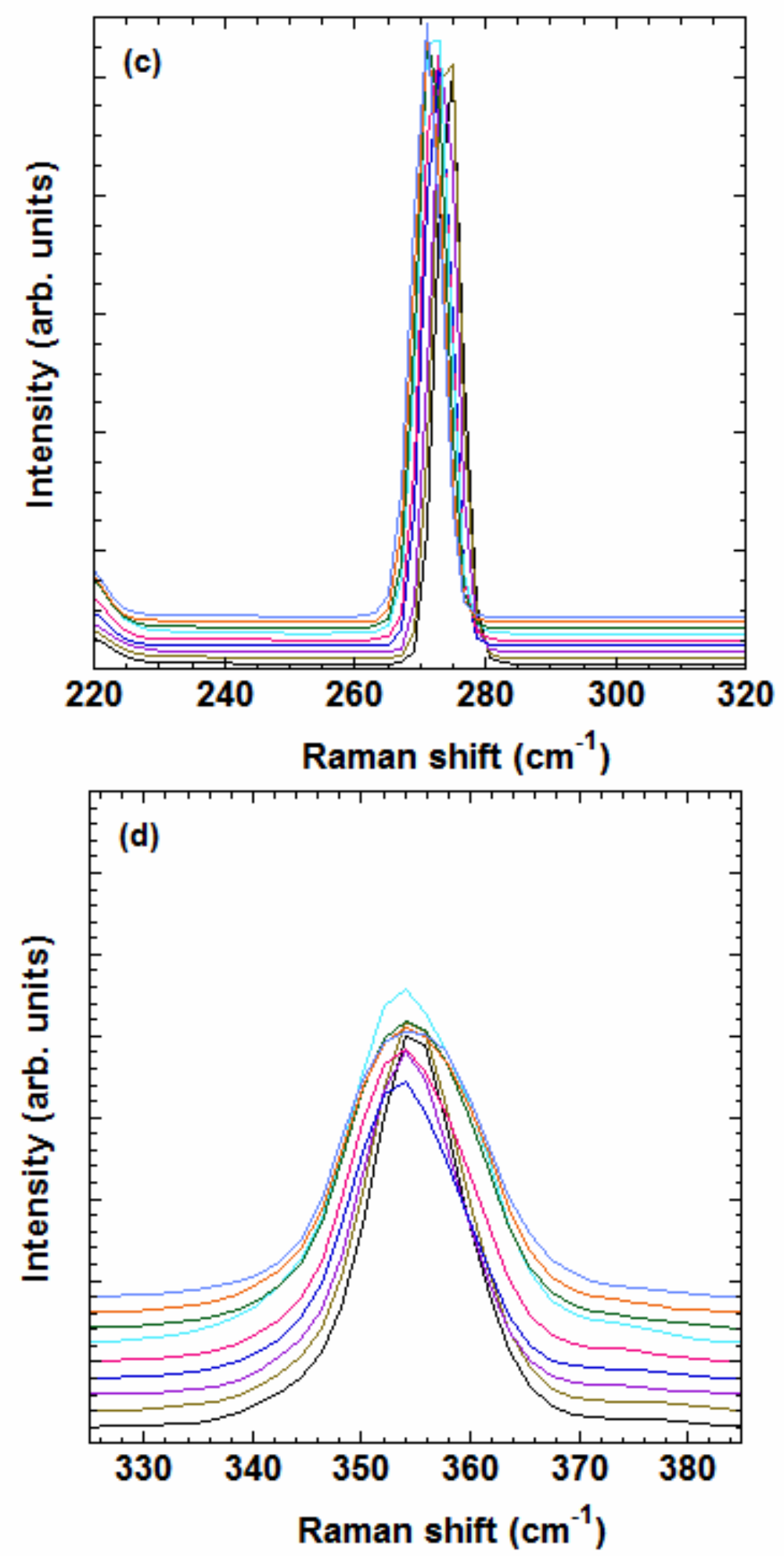

Figure 1 

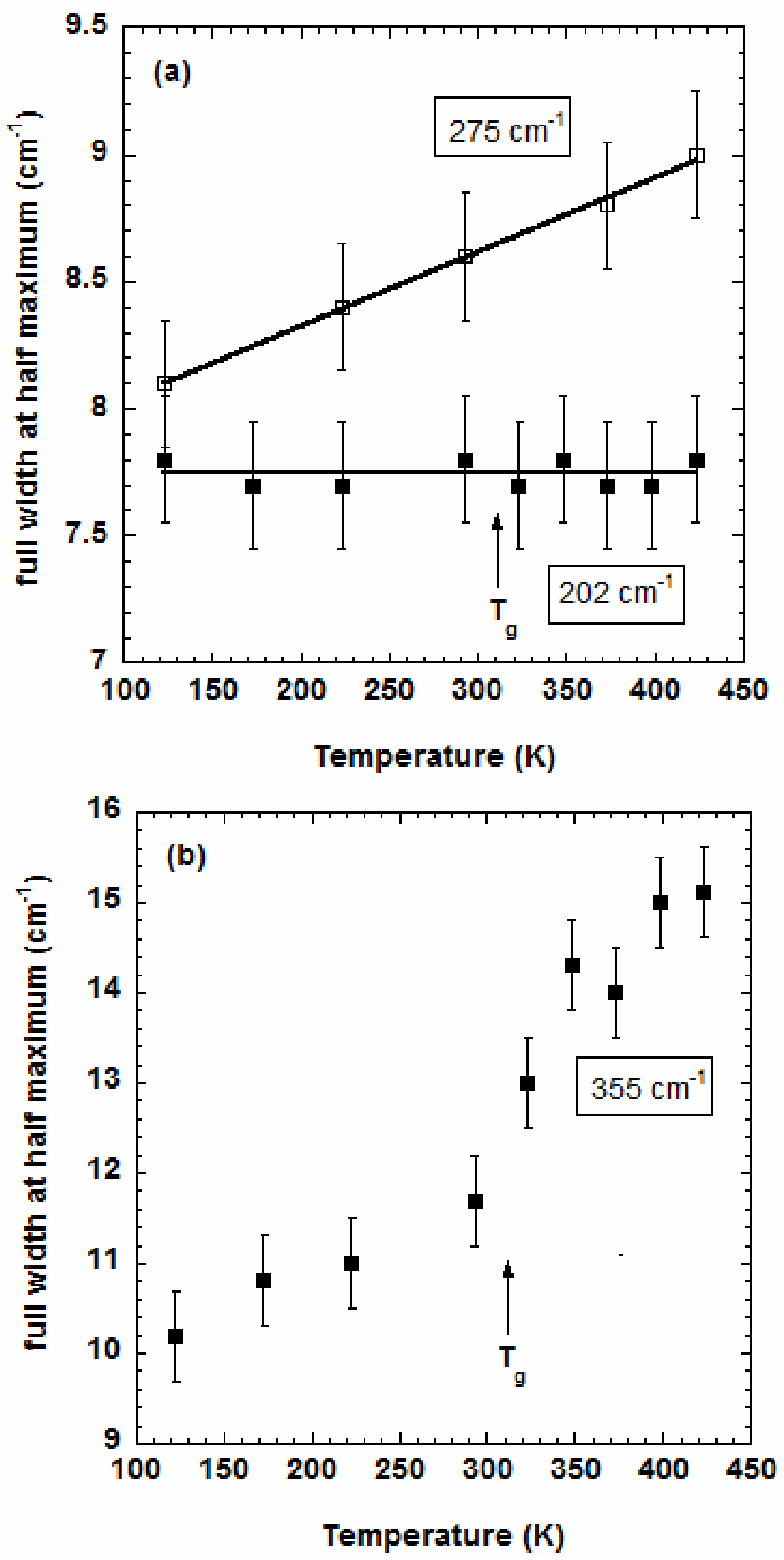

Figure 2 

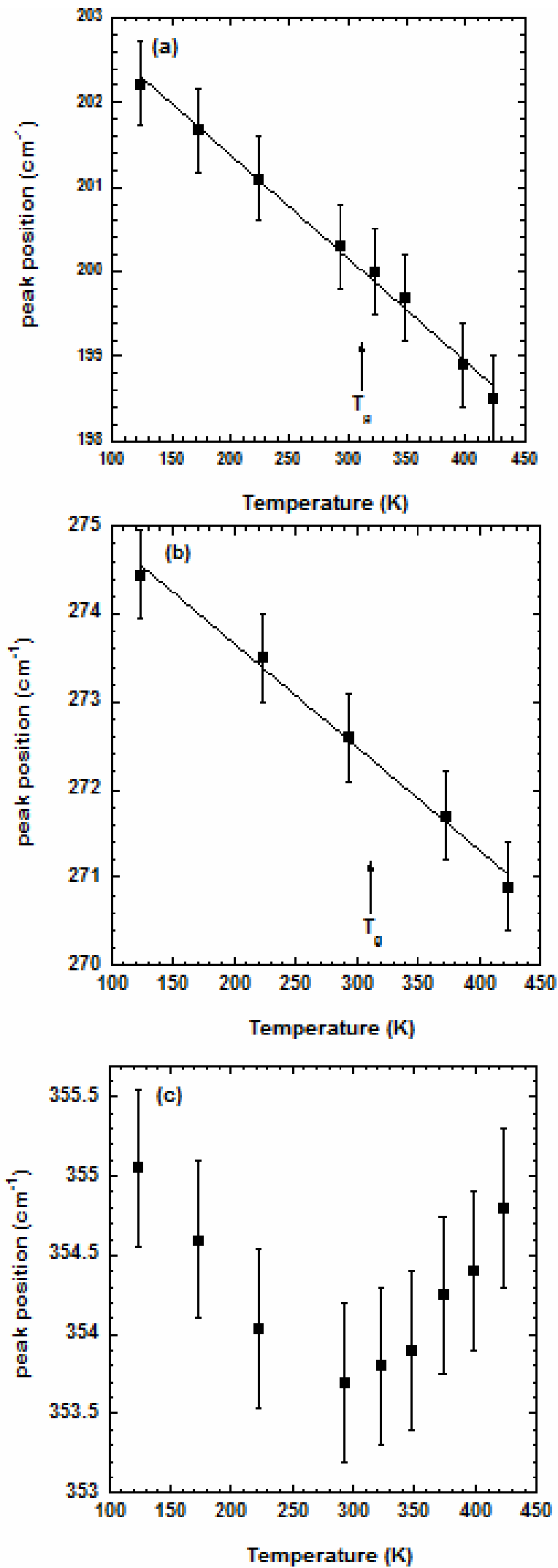

Figure 3 